\title{
The use of a cone calorimeter for the preliminary determination of the extinguishing efficiency of bulk materials
}

\author{
Bernard Król ${ }^{1, *}$, Marek Konecki ${ }^{1}$, Mariusz Klakus ${ }^{2}$, Barbara Ościłowska ${ }^{1}$ \\ ${ }^{1}$ The Main School of Fire Service, Faculty of Fire Safety Engineering, 52/54 Slowackiego St., 01-629 \\ Warsaw, Poland \\ ${ }^{2}$ State Fire Brigade, 87-300 Brodnica, Poland
}

\begin{abstract}
Determining the extinguishing effectiveness of extinguishing agents against solid fires is difficult. This is mainly due to the large variety of combustible materials. These difficulties also concern the determination of the effectiveness of various extinguishing agents for gas, fat and metal fires. However, extinguishing tests for liquid fires are defined very precisely. The tests of the effectiveness of powder materials, such as extinguishing powders, are based on relatively simple and methods used to extinguishers testing. It does not eliminate the very significant impact of the human factor on the test effect. A cone calorimeter was used for comparative evaluation of the extinguishing efficiency of bulk materials. For this purpose, bulk materials e.g. salt, sand and dry chemicals were applied to the surface of standard pine wood samples and the differences in their flammability were examined. Based on the analysis of HRR, weight loss curves and time to achieve flame combustion, it was found that a cone calorimeter can be used to pre-assess the effectiveness of extinguishing the dry powders and dry chemicals for solid fires. There were clear differences between chemical and only physical effects.
\end{abstract}

\section{Introduction}

There are in fire protection many extinguishing agents, which were used primarily in handheld firefighting equipment, fixed fire extinguishing systems and used by fire brigades. Manufacturers of extinguishing agents offer a wide range of products with different composition, properties and price. The selection of the best extinguishing agent plays a key role in minimizing fire losses. This resulted in the need to develop tests and classifications assessing their most important parameters, which affect the functional characteristics. The most important feature of extinguishing agents is an ability to quick and effective extinguishing of specific fire, the so-called extinguishing efficiency.

Wood and wood-based materials not subjected to any treatment improving their fire properties are flammable. In fire conditions they decompose relatively easy, are ignited and

\footnotetext{
*Corresponding author: bkrol@sgsp.edu.pl
} 
the flames spread quickly. This is mainly due to the structure of cellulose materials. Wood as an organic material has in its composition a high percentage of oxygen atoms. The oxidizer content in the fuel creates favorable conditions for initiating and development the combustion reaction [1]. Wood, depending on the type, has different physical properties and a different chemical composition. The key physical properties affecting flammability include, among others wood specific weight, moisture content, porosity, hardness, thickness and form. The chemical composition of wood and its structural composition also determine important fire characteristics such as: specific heat, thermal conductivity, heat capacity and heat of combustion [2].

One of the ways to stop the burning of wood is the use of fire retardants also known as flame retardants or combustion inhibitors. These are chemicals used as an additive to materials that increase their fire resistance by delaying the moment to ignite, reducing the intensity of combustion and reducing the rate of spread of flames over the surface [3]. The preparations used to protect wood against fire are usually simple chemical compounds in the form of salts. The most commonly used include: chlorides, borates, phosphates, sulphates, silicates, carboxylic acids, some hydroxides, acid anhydrides, nitriles, aldehydes and their derivatives. Retardants are also fire extinguishing agents [4]. Fire retardants are intended for forest firefighting in the form of liquid concentrates, gels or in the form of water-soluble powders. Their composition consists of inorganic salts that determine the impact on the combustion process and substances improving their usable properties such as: thickeners that increasing solution viscosity, dyes, corrosion inhibitors, preservatives and other compounds that resist the high temperature. The active substances are phosphate compounds: ammonium dihydrogen phosphate, ammonium hydrogen phosphate and polyphosphates [4].

Extinguishing powders are extinguishing media in the form of fine-grained solids. Most often these are simple inorganic salts with a composition depending on the purpose and quality of the extinguishing powders. Extinguishing powders can be divided into three basic groups marked with the letters of fire classes (ABC, BC, D). The suitability of a powder to extinguish a particular fire classes is determined by its chemical composition. The influence of extinguishing powders on the combustion process consists of:

- heat absorption due to heating of grains,

- heat absorption due to decomposition or phase transitions of a solid,

- $\quad$ isolation of combustible material from the oxidizer by creating a physical barrier,

- dilution of the combustion zone by the products of thermal decomposition and evaporation,

- flame inhibition effect on the surface of dry powder and its decomposition products,

- inhibition effect against no flame combustion process,

- $\quad$ increasing the amount of heat emission [5].

Extinguishing powders are small grains that, like any solid introduced into the flame in the form of a cloud, disturb the combustion process. This is a typically physical reaction involving the absorption of heat by the particles of extinguishing powder. This type of effect on combustion reactions is not only exhibited by specialized extinguishing powders, but by any particulate solids. The requirement is a very large specific surface, which allows for quick heating in the flame. The substances that can be used as fire extinguishing powders with only physical activity include, among others rock dust, limestone or aluminum oxide. Due to the lack of chemical effects, they have not found widespread use as a fire fighting agent due to their low extinguishing efficiency. The mass concentration need to extinguish the flame with these solids reaches $1-2 \mathrm{~kg} / \mathrm{m}^{3}$, whereas for extinguishing powders not exceed $0.1-0.5 \mathrm{~kg} / \mathrm{m}^{3}$. The heat absorption can also take place during the decomposition or phase transformation of extinguishing powders. Powders there are used in fire protection to extinguishing the fires, however, have a negligible ability to cool the 
surface of the material. This fact is important for the use of extinguishing powders for class A fires. The use of powders gives the positive and visible effect only in the first phase of fire, when the flame did not penetrate inside the burning material [6].

Determination of extinguishing effectiveness is the subject of numerous scientific works. There are many research methods and tests assessing the quality and effectiveness of extinguishing agents. Most of these tests are based, as a rule, on a visual statement of the end of the flame combustion of a specific material (from solids the most common is wood). However, they do not take into account measurements of fire parameters and changes in the combustion process. One of the applied in practice methods is based on the introduction of the extinguishing powder in the form of a cloud to the test fire. The disadvantage of this test method is the device (most often the extinguisher) and the operator's experience. There is also a difficulty in ensure the reproducibility of the test conditions. In this test conditions the assessment is based on a visual valuation only and the extinguishing efficiency is usually indicated as a fire test size (fire rating). This approach is used in the EN 615, EN 37 , ISO 7165 and many others $[7,8,9]$.

The most accurate method of laboratory examinations of extinguishing effectiveness is the method developed by W. Hoffmann [10]. The principle of this test is based on the fact that the combustion process is determined by the rate of flame spread. Introducing of the extinguishing powder into the flame results in a change in the rate of flame spread depending on the amount of powder applied and its extinguishing effectiveness. Determination of the extinguishing efficiency is based on the assessment of the rate of flame propagation. This method is only used for flame burning, not for class A fires and smoldering combustion. Similar studies were carried out at US Naval Research Laboratory [11].

\section{Methods and materials}

The idea of the research is an attempt to use a cone calorimeter as a tool for a comparative assessment of the effectiveness of extinguishing of dry powders and other bulk materials that are applied on the solid surface (class A fires). Cone calorimeter does not give the possibility of application of the agent in the form of a cloud or during the measurements on the surface of the burning material. This unable to create the real fire behavior and firefighting conditions. It also limits the types of extinguishing agents that can be tested with this method to those that can be applied on the surface and keep their form until the test begins (they will not melt and evaporate). These conditions are met by dry chemical powders. To do that, measurements of fire characteristics of pine wood covered with selected extinguishing agents subjected to a heat flux imitating fire conditions were made. The test material include substances in the form of powder, fine solids that can be used to interrupt the combustion process, such as: commercial ABC dry powder, commercial BC dry powder, standard components of extinguishing powders: ammonium dihydrogen phosphate - $\left(\mathrm{NH}_{4}\right) \mathrm{H}_{2} \mathrm{PO}_{4}$, sodium bicarbonate - $\mathrm{NaHCO}_{3}$, ammonium sulphate - $\left(\mathrm{NH}_{4}\right)_{2} \mathrm{SO}_{4}$, as well as sand and sodium chloride $-\mathrm{NaCl}$.

The tests were carried out using a cone calorimeter in accordance with the guidelines specified in the ISO 5660-1: 2002 Standard [12, 13]. During the measurements were recorded, among others, the following parameters:

- time to ignition,

- intensity of HRR heat release.

As a burning material pine wood boards with a density of $350 \pm 10 \mathrm{~kg} / \mathrm{m}^{3}$ (dimensions: $100 \mathrm{~mm} \times 100 \mathrm{~mm}$ and $20 \mathrm{~mm}$ ) were used. Wood samples were covered with a layer of powdered solid of mass $3.5 \mathrm{~g}$. For the purposes of the tests, it was assumed that the application of extinguishing agents increases the mass of the wood samples. Due to the 
differences in the distribution of the solid substance and the specific density of the particles, it was assumed that wood samples after application of the test material do not increase their dimensions. Samples were subjected to an external thermal radiation of $50 \mathrm{~kW} / \mathrm{m}^{2}$ and piloted ignition, simulating thermal exposure of the beginning of the second phase of fire.

\section{Results and discussion}

In Figure 1 different HRR curves can be observed. For each of the examined samples, analogous curves can be specified, which, however, differ in the time and the values. At the initial stage of the tests, a small increase in the HRR value were observed, followed by a rapid increase in the heat release intensity referred to as the first peak. This effect is related to the ignition of volatile thermal decomposition products. From the of individual HRR curves illustrated in Figure 1, it is possible to show a different impact of the extinguishing agents on the thermal decomposition and ignition. For unprotected wood sample, the first peak is reached at the earliest, already after about 20 seconds from the beginning of test, with a rapid jump in growth to a high value of heat release rate reaching about $1.7 \mathrm{~kW}$. A similar first peak can be observed for extinguishing preparations of physical activity: sand and sodium chloride (table salt), however, in these cases, the abrupt increase in HRR occurs after $30 \mathrm{~s}$. Protection of wood samples with dry chemical powders (ABC and BC) or compounds of dry powders significantly prolongs the time to achieve flame burning. Pine wood with ammonium phosphate and sodium bicarbonate causes a delay in the increase in the HRR to about $60 \mathrm{~s}$. BC type extinguishing powder extends the time to reach the first peak to about $80 \mathrm{~s}$ and $\mathrm{ABC}$ powder extinguishing powder to about $90 \mathrm{~s}$. For ammonium sulphate, it was not until 100 seconds after exposure to high temperature that the first peak was observed. This proves that extinguishing products with purely physical performances do not reduce the intensity of gaseous combustion products and the initiation of the flame burning process.

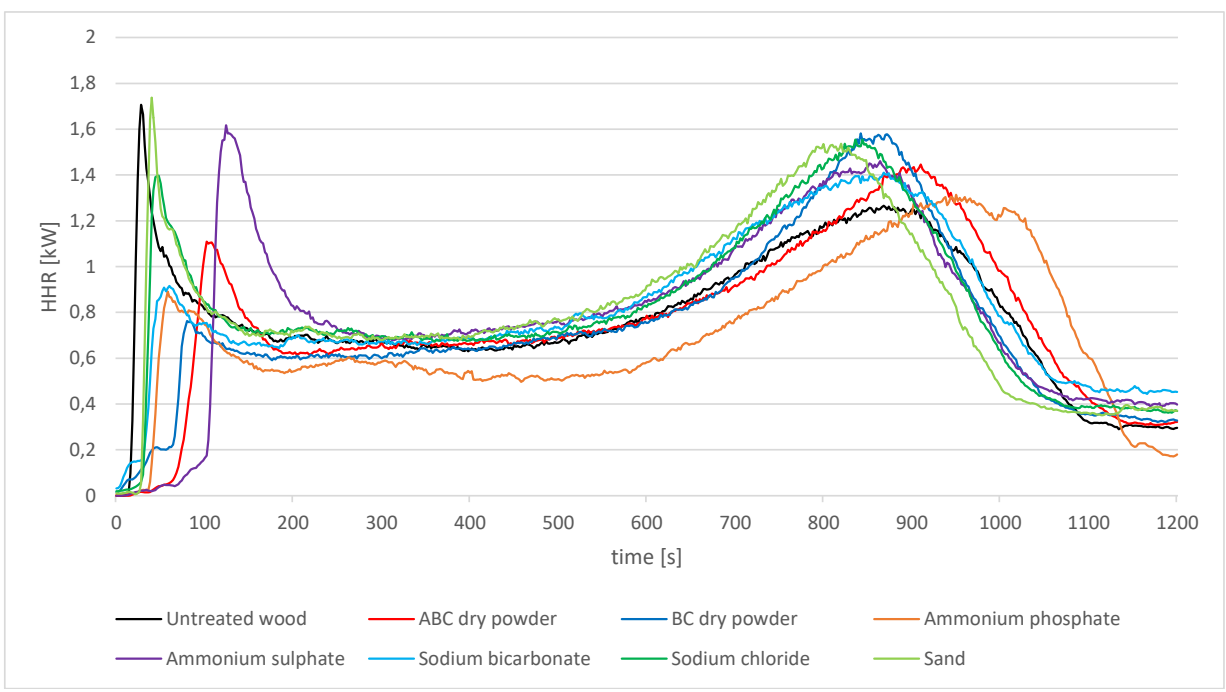

Fig. 1. Heat release rate of wood samples without and with the cover of different extinguishing agents.

Table 1 shows the time to achieve flame combustion that is in coincidence with the time of the first peak on HRR curves analyzed above. Based on the data included in Figure1 and Table 1, it can be concluded that wood with a thin layer of extinguishing agent reduces the 
intensity of generation of thermal decomposition products and delays the initiation of flame burning reactions. Substances with physical activity show the lower impact. The layer of the kitchen salt or sand only slightly increases the time to ignition. The most effective effect in this respect was demonstrated by the ammonium sulphate layer. This is the component of the ABC powders, which, like the BC type powder, significantly delayed the initiation of the flame burning process. Other chemical compounds: ammonium phosphate and sodium bicarbonate slowed down the generation of volatile combustion products with a less extent.

Table 1. Time to ignition and $\mathrm{HRR}_{\max }$ at first peak of untreated wood and wood with the cover of different extinguishing agents.

\begin{tabular}{|c|c|c|}
\hline Preparation & Time to ignition [s] & $\mathbf{H R R}_{\max }[\mathbf{k W}]$ \\
\hline Untreated wood & 20 & 1,71 \\
\hline Sand & 28 & 1,74 \\
\hline Sodium chloride (table salt) & 30 & 1,40 \\
\hline Sodium bicarbonate & 32 & 0,92 \\
\hline Ammonium phosphate & 38 & 0,90 \\
\hline BC dry powder & 50 & 0,75 \\
\hline ABC dry powder & 64 & 1,11 \\
\hline Ammonium sulphate & 100 & 1,62 \\
\hline
\end{tabular}

Based on the results presented in Figure 1, it was observed that for different samples the $\mathrm{HRR}_{\max }$ value is achieved in other combustion phases (first or second peak). Therefore the analysis includes the maximum values of heat release rates only for the first peak. Table 1 is a summary of the maximum values of the heat release rate obtained in the first peak. Only the peak value of HRR during the first peak was analyzed, because the action of the extinguishing agents applied was considered only in terms of the possibility of extinguishing a fire. Among the samples tested, the highest value of this parameter was noted for the unprotected sample, however, similar values were observed for samples covered with sand and ammonium sulphate. These substances do not therefore reduce the rate of heat release in the initial combustion phase. A significant reduction in $\mathrm{HRR}_{\max }$ for the first peak was for samples protected with $\mathrm{ABC}$ and $\mathrm{BC}$ powders, ammonium phosphate and sodium bicarbonate, with the last extinguishing agent ( $\mathrm{BC}$ powder) being the most effective in this respect.

After a sudden increase in the heat release rate occurring at the first peak, the HRR value decreases and stabilizes at a certain level for the time specified for each test. This effect is related to the formation of a charred layer on the surface of the wood, limiting the emission of volatile thermal decomposition products feeding the flame combustion zone. In Figure 1, a similar run of curves of HRR values from about $200 \mathrm{~s}$ to $600 \mathrm{~s}$ can be observed. After a period of stabilization, the HRR value increased again, reaching the maximum in the second peak. This effect is associated with the destruction of the surface structure of wood. The second peak is different for every probes, both in terms of time of occurrence and maximum values achieved. It can be noted, however, that for all curves, the second growth in the heat release intensity does not proceed as rapidly as the first. Comparing the run of individual curves in Figure 1, it can be seen that the sample with ammonium phosphate has reached the second peak at the latest. A significant increase in time to reach the second peak is also observed for the sample protected with an ABC powder containing ammonium phosphate. It can mean an effect on the no flame combustion and need to analyze the second peak in time and value. The run and time of appearance of the second peak for the others HRR curves is similar. This indicates that BC type extinguishing powder, sodium bicarbonate, ammonium sulphate, sand and kitchen salt do not affect the reinforcement of the wood surface structure during smoldering combustion. After HRR reaching the second peak, the intensity of heat release decreases due to the decreasing 
amount of combustible material. The gentle and small variability of HRR changes in the second peak indicates that this fragment of the HRR curve make a problem with assess the extinguishing efficiency. Consequently, also the average HRR value may not apply.

\section{Conclusions}

The research was to show that through standard measurements in a cone calorimeter, the effectiveness of the extinguishing agents can be determined. 7 different substances were used in the tests that can be used as extinguishing agents for surface application. They represent groups of commonly used extinguishing agents, which are characterized by different physical properties and behave in different ways under the influence of high temperature. Covering the wood surface with some solid extinguishing agents improves its fire properties.

During the tests, an extended time was recorded to initiate flame combustion and changing of the rate of heat released. Graphs of the heat rate of the analyzed samples showed a standard for the run, in which two peaks can be specified. The first peak is associated with an increase in HRR due to the initiation of flame combustion, the second peak occurs after the interruption of the homogeneity of the charred layer and the increase of the surface undergoing the thermal decomposition. Samples protected with some extinguishing agents showed differences in the run of heat release curves. The graphs for each of the samples are different in terms of time of occurrence as well as values registered in characteristic combustion phases.

The examined extinguishing agents showed different effects on the combustion process. Fire extinguishing agents that only physically react to the combustion process have had little effect on fire behavior. The assessment of the extinguishing media efficiency and their impact on the course of the HRR curve requires careful analysis - in particular, the impact on most important values: time to ignition and $H_{R R}$ max.

It is not possible to imitate in cone calorimeter the real fire conditions. However, these studies provided comprehensive results of many fire parameters that characterize the behavior of material subjected to high heat flux. They allowed to observe a clear impact of tested extinguishing agents on the fire properties of wood. It has also been shown that individual extinguishing agents influenced the various stages of combustion to varying degrees. This information confirms the sense of using a cone calorimeter to evaluate the extinguishing efficiency.

\section{References}

1. R. Curkeet, Wood combustion basics, (EPA Workshop, 2011)

2. Q. Xu et al., Constr. Build. Mater. 96, 416 (2015)

3. S.L. LeVan, J.E. Winandy, Wood. Fiber Sci. 22(1), 113 (1990)

4. A. Giménez, et al., Int. J. Wildland Fire 13, 1 (2004)

5. R. Friedman, Principles of Fire Protection Chemistry and Physics (NFPA, 1998)

6. G. Geyer, Equivalency Evaluation of Firefighting Agents and Minimum Requirements at US Air Force Airfields - Final Report (US Department of Transportation, 1982)

7. EN 615:2009

8. EN 3-7:2004+A1:2007

9. ISO 7165:2017 
10. W. Hoffmannn, Development of a laboratory test method for determining the extinguishing efficiency of dry powders, Report No. 18 and 20 (Universtät Karlruhe, 1971)

11. G. Fischer, J.T. Leonard, Effectiveness of fire extinguishing powders based on small scale suppression tests, (US Naval Research Laboratory, Washington 1995)

12. ISO 5660-1:2002

13. V. Babrauskas, R.D. Peacock, Fire Saf. J. 18, 255, (1992) 